\title{
Investigation of the surrogate-reaction method via the simultaneous measurement of gamma-emission and fission probabilities
}

\author{
B. Jurado ${ }^{1, \text { a }}$, P. Marini ${ }^{1}$, L. Mathieu ${ }^{1}$, M. Aiche ${ }^{1}$, S. Czajkowski $^{1}$, I. Tsekhanovich ${ }^{1}$, L. Audouin ${ }^{2}$, G. Boutoux ${ }^{3}$, \\ D. Denis-Petit ${ }^{4}$, M. Guttormsen ${ }^{5}$, G. Kessedjian ${ }^{6}$, M. Lebois ${ }^{2}$, V. Méot ${ }^{4}$, A. Oberstedt ${ }^{7}$, S. Oberstedt ${ }^{8}$, O. Roig $^{4}$, O. Sérot $^{9}$, \\ L. Tassan-Got ${ }^{2}$, and J.N. Wilson ${ }^{2}$ \\ ${ }^{1}$ CENBG, CNRS/IN2P3-Université de Bordeaux, Chemin du Solarium, BP. 120, 33175 Gradignan, France \\ 2 IPN d'Orsay, Bâtiment 100, 15 rue G. Clemenceau, 91406 Orsay Cedex, France \\ 3 CELIA, 43 rue Pierre Noailles, 33405 Talence Cedex, France \\ 4 CEA DAM DIF, 91297 Arpajon, France \\ 5 Department of Physics, University of Oslo, 0316 Oslo, Norway \\ ${ }^{6}$ LPSC, Université Grenoble-Alpes, CNRS/IN2P3, 38026 Grenoble Cedex, France \\ 7 Fundamental Fysik, Chalmers Tekniska Högskola, 41296 Göteborg, Sweden \\ 8 EC-JRC, IRMM, Retieseweg 111, 2440 Geel, Belgium \\ ${ }^{9}$ CEA-Cadarache, DEN/DER/SPRC/LEPh, 13108 Saint-Paul-lez-Durance, France
}

\begin{abstract}
We present the results of two experiments where we have measured for the first time simultaneously the fission and gamma-decay probabilities induced by different surrogate reactions. In particular, we have investigated the ${ }^{238} \mathrm{U}(\mathrm{d}, \mathrm{p}),{ }^{238} \mathrm{U}\left({ }^{3} \mathrm{He}, \mathrm{t}\right)$ and ${ }^{238} \mathrm{U}\left({ }^{3} \mathrm{He},{ }^{4} \mathrm{He}\right)$ reactions as surrogates for the neutron-induced $n+{ }^{238} \mathrm{U}, \mathrm{n}+{ }^{237} \mathrm{~Np}$ and $\mathrm{n}+{ }^{236} \mathrm{U}$ reactions, respectively. In the region where gamma emission, neutron emission and fission compete, our results for the fission probabilities agree fairly well with the neutron-induced data, whereas our gamma-decay probabilities are significantly higher than the neutroninduced data. The interpretation of these results is not obvious and is discussed within the framework of the statistical model with preliminary results for calculated spin-parity distributions populated in surrogate reactions. We also present future plans for surrogate-reaction studies in inverse kinematics with radioactiveion beams at storage rings.
\end{abstract}

\section{Introduction}

Neutron-induced cross sections of short-lived nuclei are key input information for applications in nuclear technology and for nuclear astrophysics. However, in many cases the measurement of these cross sections is very difficult or even impossible, due to the radioactivity of the targets involved. The surrogate-reaction method can help to overcome these difficulties. This indirect method consists in using an alternative (or surrogate) reaction to produce the same decaying nucleus $A^{*}$ as the one formed in the neutron-induced reaction of interest. The decay probability induced by the surrogate reaction is measured, and the desired neutron-induced reaction is "simulated" by multiplying the measured decay probability by the calculated cross section for the formation of a compound nucleus after neutron absorption. For more details on the surrogate-reaction method, see [1]. The usefulness of the surrogate method is that in some cases it is possible to find a surrogate reaction where the needed target is stable or less radioactive than the target of the corresponding neutron-induced reaction.
For the surrogate-reaction method to give cross section results in agreement with those obtained in direct measurements, several conditions have to be fulfilled [1]. First, both the neutron-induced and the surrogate reactions must lead to the formation of a compound nucleus. In that case the decay of nucleus $A^{*}$ is independent of the entrance channel, and the reaction cross section can be factorized into the product of the compound-nucleus formation cross section and the decay probability. The second condition is that the decay probability measured in the surrogate reaction has to be equal to the decay probability in the neutron-induced reaction. This is the case in at least two limiting situations: if the angular momentum $(J)$ and parity $(\pi)$ distributions populated in the neutron- and transferinduced reactions are the same, or if the decay probability of the compound nucleus is independent of its angular momentum and parity, which is the so-called WeisskopfEwing limit. Since for most surrogate reactions it is not yet possible to determine the populated $J^{\pi}$ distribution [1], the validity of the surrogate method has to be verified "a posteriori", by comparing the obtained results with well known neutron-induced data.

Surrogate-reaction studies performed in the last decade have shown that fission cross sections obtained via the surrogate-reaction method are generally in good

a e-mail: jurado@cenbg.in2p3.fr

(C) The Authors, published by EDP Sciences. This is an Open Access article distributed under the terms of the Creative Commons Attribution License 4.0 (http://creativecommons.org/licenses/by/4.0/). 
agreement with the corresponding neutron induced data up to equivalent neutron energies of $10 \mathrm{MeV}$, see e.g. [2] and other examples included in [1]. However, discrepancies as large as a factor 10 have been observed when comparing radiative-capture cross sections of rareearth nuclei obtained in surrogate and neutron-induced reactions [3,4]. These significant differences have been attributed to the higher angular momenta populated in the surrogate reaction. At excitation energies close to the neutron separation energy $S_{\mathrm{n}}$, neutron emission is very sensitive to the angular momentum of the decaying nucleus $A^{*}$, as only the ground state and the first excited states of the residue nucleus $A-1$ can be populated. When the angular momentum of $A^{*}$ is considerably higher than the angular momentum of the first states of $A-1$, neutron emission is hindered and the nucleus $A^{*}$ predominantly decays by gamma emission, which is the only open decay channel [4]. Similarly to the situation at energies close to the ground state, the energy region close to the fission barrier is also characterized by a low density of states and a significant dependence of the fission probability on the angular momentum is expected by theory [1]. Therefore, it is surprising that the spin/parity mismatch between the surrogate and neutron-induced reactions has no major impact on the measured fission probabilities.

To shed light into this puzzling observation, it is first of all necessary to demonstrate the much weaker sensibility of the fission probability to angular momentum, by simultaneously measuring fission and gamma-decay probabilities for the same nucleus at the same excitation energy. This has never been done before and was the object of a campaign of measurements we performed over the last years. We first investigated the ${ }^{238} \mathrm{U}(\mathrm{d}, \mathrm{p})$ reaction at the Oslo Cyclotron Laboratory. The details of the experimental set-up and the procedure to infer the gamma-emission and fission probabilities are described in a very recent publication [5]. However, as shown in [5], the deuteron breakup complicates significantly the interpretation of our results. For this reason we performed a measurement at the Tandem Accelerator of Orsay, with a ${ }^{3} \mathrm{He}$ beam on a ${ }^{238} \mathrm{U}$ target, to investigate the ${ }^{238} \mathrm{U}\left({ }^{3} \mathrm{He}, \mathrm{t}\right)$ and ${ }^{238} \mathrm{U}\left({ }^{3} \mathrm{He},{ }^{4} \mathrm{He}\right)$ reactions as surrogates for the neutron-induced $n+{ }^{237} \mathrm{~Np}$, and $n+{ }^{236} U$ reactions, respectively [6]. These surrogate reactions are not affected by breakup effects [1], and the associated neutron-induced data on fission and capture cross sections are of good quality.

\section{Experiment at the tandem accelerator of orsay}

\subsection{Experimental setup}

The Tandem accelerator of Orsay provided a ${ }^{3} \mathrm{He}$ beam of $24 \mathrm{MeV}$ energy with an intensity of about 20 enA. The setup is sketched in Fig. 1. It consisted of a vacuum chamber housing the ${ }^{238} \mathrm{U}$ target, two position-sensitive particle telescopes centered at 140 degrees and a fission detector. The chamber was surrounded by two types of gamma detectors: six high-purity germanium detectors and four $\mathrm{C}_{6} \mathrm{D}_{6}$ liquid scintillators. The telescopes were used to identify and measure the kinetic energies and the angles of the ejectiles. Gamma rays and fission fragments were detected in coincidence with the ejectiles.

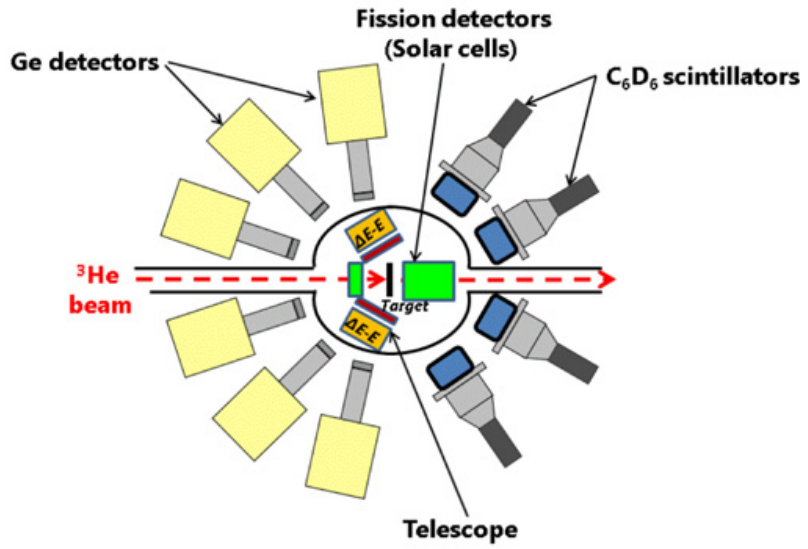

Figure 1. Schematic view of the setup used at the Tandem accelerator of Orsay for the simultaneous measurement of fission and gamma decay-probabilities.

The ${ }^{238} \mathrm{U}$ target had a thickness of $250 \mu \mathrm{g} / \mathrm{cm}^{2}$ and an isotopic purity of $99.7 \%$. A $20 \mu \mathrm{m}$ thick aluminum foil was placed in front of the telescopes to stop fission fragments. The fission detector consisted of 16 solar cells surrounding the beam in a very compact geometry close to the target. Each side of the detector, $40 \mathrm{~mm}$ long, was composed of two cells. The length of the cells varied from one detector side to the other in order to cover different mean polar angles. The forward cells covered angles from 15 to 63 degrees and the backward cells from 110 to 150 degrees in steps of few degrees. This segmentation allowed us to measure the fission-fragment angular anisotropy in the center of mass. The geometrical efficiency of the fission detector was measured with a ${ }^{252} \mathrm{Cf}$ source of known activity and amounted to $\sim 58 \%$. The effective fission efficiency, which accounts for the fission-fragment angular anisotropy in the center of mass and the forward focusing of the fission fragments due to kinematic effects, was obtained with a Monte-Carlo simulation, previously validated with the data obtained with the ${ }^{252} \mathrm{Cf}$ source. The effective fission efficiency amounted to about $65 \% . \mathrm{C}_{6} \mathrm{D}_{6}$ scintillators are very well adapted for determining the gamma-decay probability, as shown in [4]. One important advantage of the $\mathrm{C}_{6} \mathrm{D}_{6}$ is that they allow one to distinguish gamma-rays from neutrons via pulse-shape discrimination. The Ge detectors were used to measure the probabilities of populating selected gamma transitions, but the results will not be discussed here.

\subsection{Data analysis}

The decay probability in the outgoing channel $\chi$ of the decaying nucleus $A^{*}$ produced in a transfer reaction $X(y, b) A^{*}$ can be obtained as:

$$
P_{\chi}\left(E^{*}\right)=\frac{N_{\chi}^{C}\left(E^{*}\right)}{N^{S}\left(E^{*}\right) \cdot \varepsilon_{\chi}\left(E^{*}\right)}
$$

Here $N^{S}\left(E^{*}\right)$ is the so-called "singles spectrum", i.e., the total number of detected ejectiles $b$ as a function of the excitation energy $E^{*}$ of $A^{*} . N_{\chi}^{C}\left(E^{*}\right)$ is the "coincidence spectrum", corresponding to the number of ejectiles $b$ detected in coincidence with the observable that identifies the decay mode, e.g. a fission fragment or a gamma ray, 
and $\varepsilon_{\chi}$ is the associated detection efficiency. In the absence of ejectiles originating from contaminant reactions, the quantity $N^{S}\left(E^{*}\right)$ corresponds to the total number of $A^{*}$ nuclei and $N_{\chi}^{C}\left(E^{*}\right) / \varepsilon_{\chi}$ to the number of $A^{*}$ nuclei that have decayed via channel $\chi$. In this work, only the data for the ${ }^{238} \mathrm{U}(\mathrm{d}, \mathrm{p})$ reaction were affected by contaminants, see [5] for the details on their subtraction. Our excitation-energy resolution varies between $\sim 40$ and $70 \mathrm{keV}$ depending on the reaction.

The gamma-decay probability measures the likelihood that the excited nucleus $A^{*}$ decays through a gammaray cascade, i.e., that nucleus $A^{*}$ de-excites by emitting gamma rays only. Above the neutron separation energy $S_{\mathrm{n}}$ this probability decreases very rapidly with excitation energy $E^{*}$, because of the competition with the neutronemission channel. Therefore, the gamma-decay probability is typically measured up to $E_{\max }^{*} \approx S_{\mathrm{n}}+1 \mathrm{MeV}$. As described in [4], to remove the gamma rays emitted after neutron emission from the coincidence spectrum, we applied a threshold to the energy gamma-ray spectrum $E_{\mathrm{th}}=E_{\mathrm{max}}^{*}-S_{\mathrm{n}} \mathrm{MeV}$. To determine the gamma-decay probability we need to know the gamma-cascade detection efficiency $\varepsilon_{\gamma}$ of the $\mathrm{C}_{6} \mathrm{D}_{6}$ detectors. This was obtained with the EXtrapolated Efficiency Method (EXEM) described in $[7,8]$. The resulting efficiency increases by about $1 \%$ from $E^{*}=S_{\mathrm{n}}$ to $E^{*}=S_{\mathrm{n}}+1 \mathrm{MeV}$, and varies between 4 and $6 \%$ at $E^{*}=S_{\mathrm{n}}$ for all the investigated decaying nuclei.

The measurement of the gamma-decay probability at excitation energies where the fission channel is open is challenging because of the background of gamma rays emitted by the fission fragments. This background can be removed by using the expression:

$$
N_{\gamma}^{C}\left(E^{*}\right)=N_{\gamma}^{C, t o t}\left(E^{*}\right)-\frac{N_{\gamma, f}^{C}\left(E^{*}\right)}{\varepsilon_{f}\left(E^{*}\right)}
$$

where $N_{\gamma}^{C}$ is the final number of coincidences needed to evaluate the gamma-emission probability according to Eq. (1) $\left(N_{\gamma}^{C} / \varepsilon_{\gamma}\right.$ corresponds to the number of gamma cascades emitted by nucleus $\left.A^{*}\right) . N_{\gamma}^{\mathrm{C} \text {,tot }}$ is the total number of measured gamma-ejectile coincidences after application of the $E_{\mathrm{th}}$ threshold, $N_{\gamma, \mathrm{f}}^{C}$ is the number of triple fissionfragment-gamma-ejectile coincidences and $\varepsilon_{\mathrm{f}}$ is the fission detection efficiency. See [5] for more details on the correction of fission-fragment gamma rays.

\section{Results}

In the interaction of the ${ }^{3} \mathrm{He}$ beam with the ${ }^{238} \mathrm{U}$ target several transfer channels are populated. Besides the ${ }^{238} \mathrm{U}\left({ }^{3} \mathrm{He}, \mathrm{t}\right)$ and ${ }^{238} \mathrm{U}\left({ }^{3} \mathrm{He},{ }^{4} \mathrm{He}\right)$ channels, it was also possible to measure the decay probabilities of the ${ }^{238} \mathrm{U}\left({ }^{3} \mathrm{He}, \mathrm{d}\right){ }^{239} \mathrm{~Np}^{*}$ channel, whose associated neutroninduced reaction is $\mathrm{n}+{ }^{238} \mathrm{~Np}$. Note that ${ }^{238} \mathrm{~Np}$ has a half-life of only 2.1 days and there are no fission or capture cross sections data for this nucleus at fast neutron energies. The data of ${ }^{239} \mathrm{~Np}^{*}$ are of particular interest since they can be used to test the validity of the methodology described above to simultaneously infer the fission and gamma-emission probabilities. Indeed, the fission barrier of ${ }^{239} \mathrm{~Np}$ is lower than its neutron separation energy. Therefore, for this nucleus, the two main decay modes

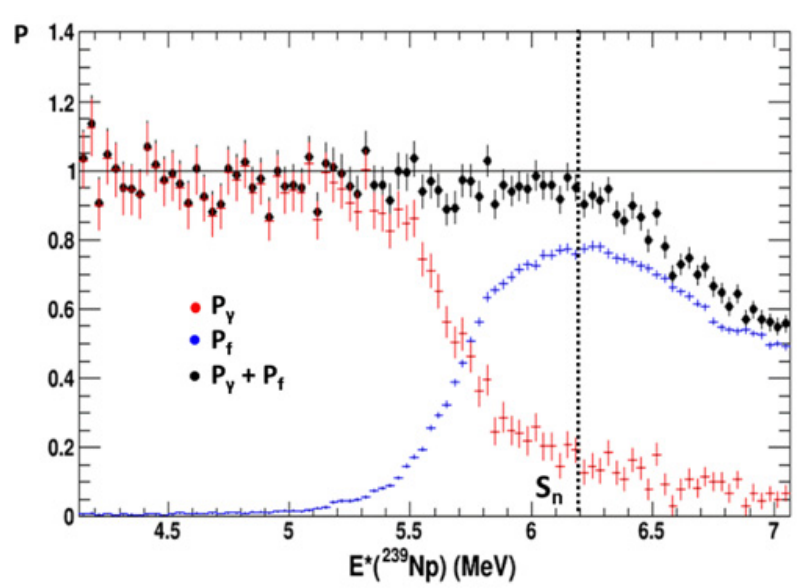

Figure 2. Preliminary results for fission (blue) and gammadecay (red) probabilities as a function of the excitation energy of ${ }^{239} \mathrm{~Np}^{*}[6]$. The sum of the fission and gamma-decay probabilities is represented by the black circles. The vertical dotted line indicates the neutron separation energy of ${ }^{239} \mathrm{~Np}$.

below $S_{\mathrm{n}}$ are fission and gamma emission, and the sum of the corresponding decay probabilities must be equal to 1 . Preliminary results for the gamma-decay and fission probabilities are shown in Fig. 2. The gamma-emission probability is first $100 \%$ and decreases when fission sets in. Both probabilities decrease at $S_{\mathrm{n}}$ because of the competition with the neutron-emission channel. We can see that the sum of the two decay probabilities is consistent with 1 below $S_{\mathrm{n}}$ within the error bars, although there is a slight underestimation between $5.5 \mathrm{MeV}$ and $S_{\mathrm{n}}$ due to the preliminary values for the gamma-cascade and fission efficiencies. Still, this is a strong proof that the used analysis procedure is correct.

Figure 3 shows preliminary results for the gammaemission and fission probabilities of the ${ }^{238} \mathrm{U}\left({ }^{3} \mathrm{He},{ }^{4} \mathrm{He}\right){ }^{237} \mathrm{U}$ reaction compared to different evaluations of the decay probabilities for the $n+{ }^{236} \mathrm{U}$ reaction. The latter have been obtained by dividing the evaluated neutron-induced cross sections by the compound-nucleus formation cross section $\sigma_{\mathrm{CN}} . \sigma_{\mathrm{CN}}$ was obtained with the phenomenological optical-model potential used in the JENDL 4.0 evaluation. The gamma-decay probability obtained with the surrogatereaction method is several times higher than the neutroninduced one over the whole excitation-energy range, although the discrepancies between surrogate and neutroninduced data decrease with excitation energy. On the other hand, the fission probability is in much better agreement with the neutron-induced data.

Figure 4 shows the gamma-decay and fission probabilities together with the corresponding neutroninduced probabilities for three different surrogate reactions in the excitation-energy region where fission, gamma and neutron emission are in competition. In all cases, the fission probability is in fairly good agreement with the neutron-induced data, whereas the gamma-decay probability obtained with the surrogate reactions is several times higher than the neutron-induced one. Note that this is also the case for the ${ }^{238} \mathrm{U}(\mathrm{d}, \mathrm{p})$ reaction (bottom panel), which intuitively appears as the reaction closest to a neutron-induced reaction. 

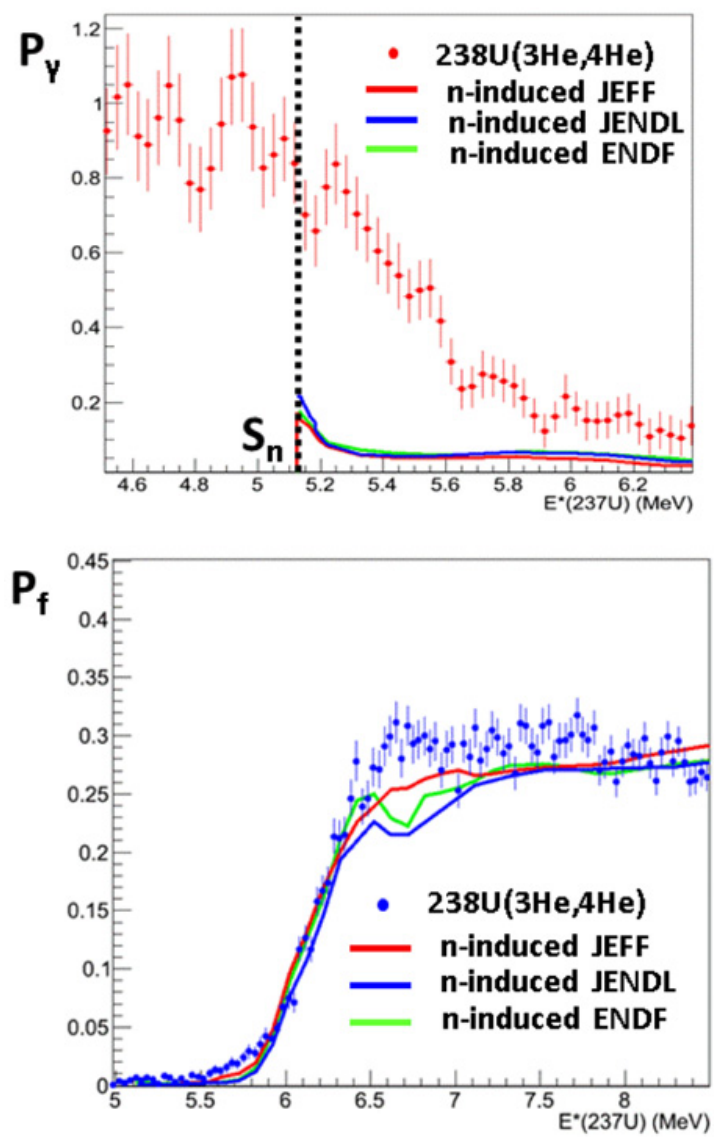

Figure 3. Preliminary gamma-decay (top) and fission (bottom) probabilities as a function of the excitation energy of ${ }^{237} \mathrm{U}[6]$ compared to the corresponding neutron-induced decay probabilities according to different evaluations. The vertical dotted line in the upper panel represents the neutron separation energy of ${ }^{237} \mathrm{U}$.

\section{Discussion}

As discussed in [5], we investigated whether we can explain the results for the ${ }^{238} \mathrm{U}(\mathrm{d}, \mathrm{p})$ reaction within the frame of the statistical model. For this purpose we used a Hauser-Feshbach Monte-Carlo code developed at the CEA/DAM laboratory with the ingredients of Ref. [9]. In the region where both decay channels compete, the calculated gamma-decay and fission probabilities show a clear dependence on the initial values of the spin and parity of nucleus ${ }^{239} \mathrm{U}^{*}$ (see Fig. 14 in [5]), demonstrating that the Weisskopf-Ewing approximation discussed in the introduction is not valid either for the gamma-emission or the fission probabilities. We used a model based on the Distorted-Wave Born approximation (DWBA) to obtain a first estimate of the average spin populated in the ${ }^{238} \mathrm{U}(\mathrm{d}, \mathrm{p})$ reaction [5]. The average spin is about $70 \%$ larger than the one populated in the neutron-induced reaction at $E^{*}=S_{\mathrm{n}}+0.5 \mathrm{MeV}$ and about $25 \%$ larger at $E^{*}=S_{\mathrm{n}}+1.5 \mathrm{MeV}$. Therefore, our results for the decay probabilities (bottom panel of Fig. 4) demonstrate that this significant change in the spin distribution has a much stronger impact on the gamma-emission probability than on the fission probability.

O. Bouland et al. [10] have performed a theoretical study of our results for the decay probabilities induced in the ${ }^{238} \mathrm{U}\left({ }^{3} \mathrm{He},{ }^{4} \mathrm{He}\right)$ reaction based on the one-dimensional
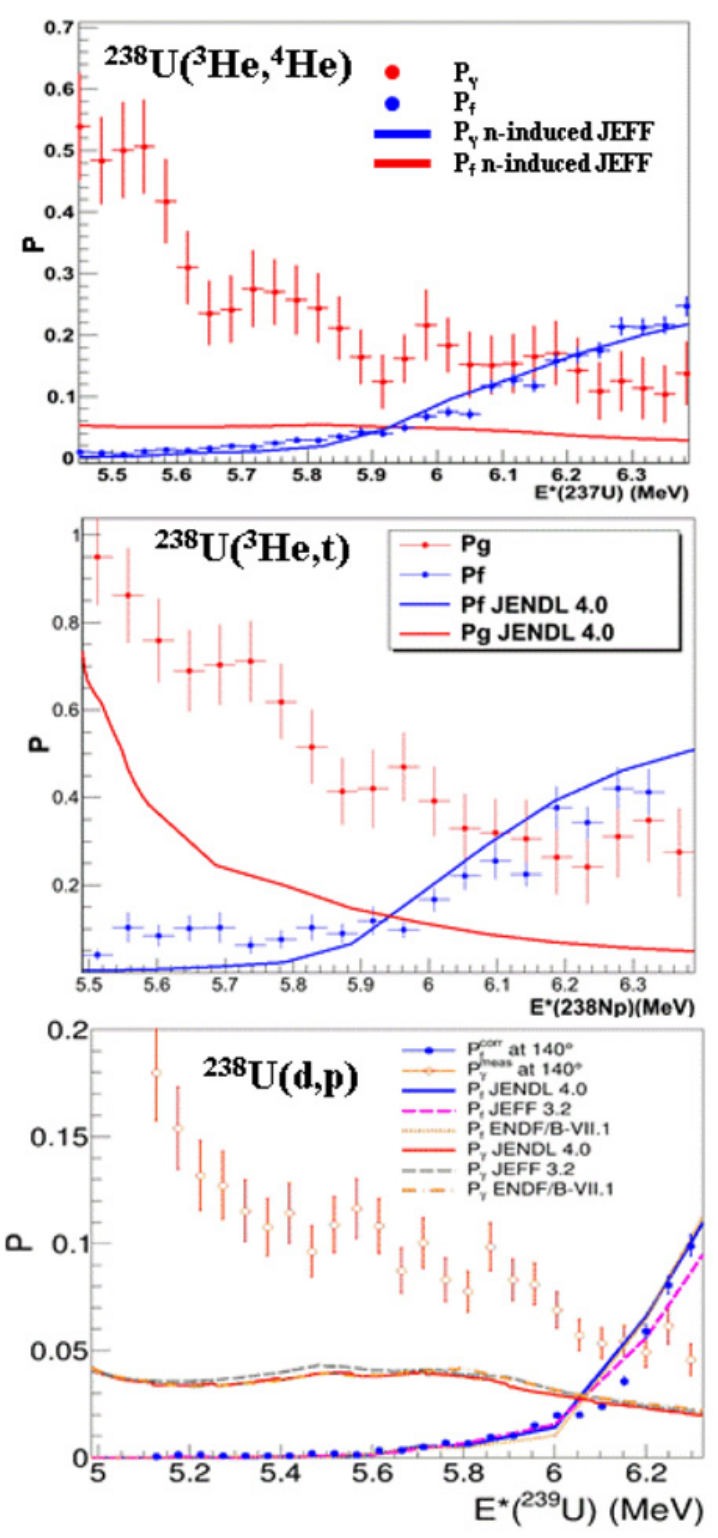

Figure 4. Preliminary gamma-decay and fission probabilities as a function of excitation energy compared to the corresponding neutron-induced decay probabilities according to different evaluations. The upper and middle figures show preliminary results for ${ }^{237} \mathrm{U}^{*}$ and ${ }^{238} \mathrm{~Np}^{*}$, respectively, measured in the Orsay experiment [6]. The bottom figure is taken from reference [5] and shows the results for ${ }^{239} \mathrm{U}^{*}$.

fission barrier extended R-matrix theory with Monte Carlo samplings of class I and class II state parameters. The preliminary calculated decay probabilities obtained in [10] show a dependence on the spin and parity for both fission and gamma emission, although, contrary to the calculations presented in [5], this dependence is much weaker for fission. As shown in [10], it is possible to reproduce our measured decay probabilities fairly well by weighting these calculated decay probabilities with the $J^{\pi}$ probability distributions of the ${ }^{238} \mathrm{U}\left({ }^{3} \mathrm{He},{ }^{4} \mathrm{He}\right)$ reaction predicted by I. Thompson and J. Escher [11]. The latter distributions are very different for positive and negative parities and significantly differ from the neutron-induced ones. The good reproduction of our fission data indicates that the agreement between the fission probabilities from surrogate and neutron-induced reactions is due to the rather 
weak dependence of the fission probability on $J^{\pi}$. The reason for the good reproduction of our gamma-emission probability is rather subtle. It lies in the fact that the values of $\boldsymbol{J}$ and $\boldsymbol{\pi}$ that are more strongly populated in the ${ }^{238} \mathrm{U}\left({ }^{3} \mathrm{He},{ }^{4} \mathrm{He}\right)$ surrogate reaction are precisely the ones that lead to similar gamma-emission probabilities, and that these probabilities are rather close to our results. This gives confidence on both the calculated $J^{\pi}$ distributions of [11] and the statistical-model calculations of [10].

The previous discussion demonstrates the interest of measuring simultaneously fission and gamma-emission probabilities for understanding the surrogate-reaction method and also for constraining statistical-model calculations.

\section{Perspectives}

In the near future we plan a new measurement with the setup described in Sect. 2.1, a ${ }^{240} \mathrm{Pu}$ target and two different beams, ${ }^{3} \mathrm{He}$ and ${ }^{4} \mathrm{He}$. Of particular interest are the ${ }^{240} \mathrm{Pu}\left({ }^{3} \mathrm{He},{ }^{3} \mathrm{He} '\right)$ and ${ }^{240} \mathrm{Pu}\left({ }^{4} \mathrm{He},{ }^{4} \mathrm{He}\right.$ ' $)$ inelasticscattering reactions, which both lead to the decaying nucleus ${ }^{240} \mathrm{Pu}^{*}$. The associated neutron-induced reaction is $\mathrm{n}+{ }^{239} \mathrm{Pu}$ for which fission and capture cross sections are well known. In addition, the density of states around the barrier of the even-even ${ }^{240} \mathrm{Pu}$ nucleus is lower than for the odd-A or odd-odd actinides studied so far, and we expect a stronger sensitivity of the fission probability to the populated angular momentum and parity. The interest of using two inelastic surrogate reactions to excite the ${ }^{240} \mathrm{Pu}$ nucleus is that spinless $\alpha$-particle projectiles excite only natural-parity states $\pi=(-1)^{J}$, whereas ${ }^{3} \mathrm{He}$ projectiles excite also non-natural-parity states [1]. Therefore, we can investigate the impact of two significantly different $J^{\pi}$ distributions.

The continuation of the investigation of the surrogatereaction method depends on the availability of appropriate targets. The targets needed for surrogate experiments are difficult to produce, as they have to be chemically pure (absence of light and heavy target contaminants) and deposited on a very thin backing to limit the pollution due to reactions on contaminants and backing. These issues can be overcome by performing the measurements in inverse kinematics with radioactive-ion beams. The GSI facility already provides actinide and pre-actinide beams over long isotopic chains. This variety of beams offers the possibility to perform the systematic studies that are still necessary for understanding the surrogate-reaction method. However, the quality of radioactive beams in terms of beam-energy definition, as well as position and energy resolution, is too low for accurate decay-probability measurements near the fission threshold or near the neutron-separation energy, where the probabilities vary rapidly with excitation energy.

We think that the best place to measure decayprobabilities in inverse kinematics is at storage rings. The possibility to measure proton-capture cross sections inside a storage ring has been recently demonstrated at the ESR (Experimental Storage Ring) at GSI [12]. This measurement represented a real technological challenge, as the detectors placed inside the ring had to stand the very demanding ultra-high vacuum conditions. This possibility opens up highly interesting opportunities for surrogate-reaction experiments. Indeed, the stored ions are cooled down in an electron cooler and have a much

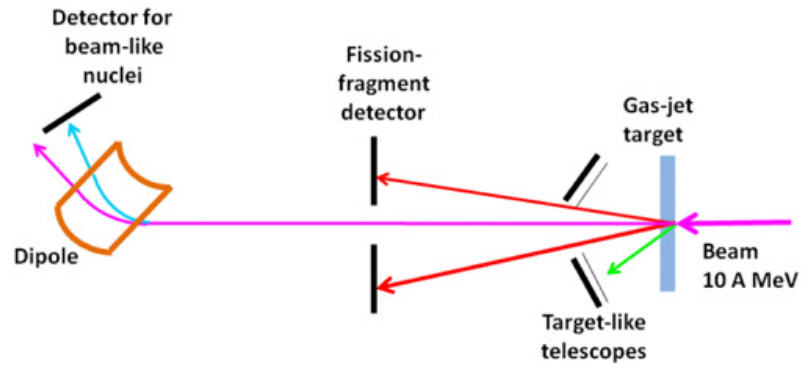

Figure 5. Schematic view of a possible in-ring set-up for the simultaneous measurement of fission and gamma-emission probabilities in inverse kinematics.

better beam quality than non-stored radioactive beams. The beam energy definition is excellent, the beam energy resolution $\triangle E / E$ is improved from typically $10^{-3}$ to about $10^{-4}$, and the beam size reduced from several $\mathrm{mm}$ to $1 \mathrm{~mm}$ diameter [13]. The stored ions interact with windowless gas-jet targets located inside the ring, for which thicknesses up to about $10^{14}$ atoms $/ \mathrm{cm}^{2}$ can be obtained for light gases as $\mathrm{H}_{2}, \mathrm{D}_{2},{ }^{3} \mathrm{He}$ and ${ }^{4} \mathrm{He}$. Contrary to a single-pass (standard) measurement, the stored ions turn with frequencies of the order of $1 \mathrm{MHz}$ (at $10 \mathrm{~A} \mathrm{MeV}$ ) and are repeatedly recycled after passing the target. Therefore, the revolution frequency leads to an increase of the effective target thickness by almost a factor $10^{6}$, which then becomes comparable with the thickness of a target foil in standard experiments. The use of windowless, pure targets represents an enormous advantage for the measurement of decay probabilities, as there will be no pollution from reactions in the target backing or in target contaminants, which is one of the most significant complications for the measurements in direct kinematics [5]. Last but not least, the stored ion beams are highly pure and it is even possible to store pure isomeric beams. This would allow us to study angular-momentum effects in a very precise way by comparing the results for the same surrogate reaction carried out with the beam in the ground state and with the beam in the isomeric state.

Figure 5 shows a sketch of a possible in-ring setup for the simultaneous measurement of decay probabilities. It consists of two position sensitive telescopes to identify the target-like residues and measure their kinetic energies and angles, and a detector to measure the fission fragments in coincidence. This part of the set-up serves to determine the fission probabilities and should be placed in a reaction chamber surrounding the gas-jet target. To measure the gamma-emission probability, the projectile-like residues are detected in coincidence with the target-like residues by a detector positioned after one of the ring dipoles. Thanks to the dipole the projectile-like residues that decay by emitting only gamma rays are separated from the elastic or inelastic scattered beam, which does not hit the detector. The equipment to detect projectile-like residues already exists at the ESR. We are now starting to work on the design of the reaction chamber around the ESR gas-jet target and on the target-like and fission-fragment detectors.

\section{Conclusions}

We have measured for the first time simultaneously fission and gamma-emission probabilities induced by the 
${ }^{238} \mathrm{U}\left({ }^{3} \mathrm{He}, \mathrm{d}\right),{ }^{238} \mathrm{U}\left({ }^{3} \mathrm{He}, \mathrm{t}\right),{ }^{238} \mathrm{U}\left({ }^{3} \mathrm{He},{ }^{4} \mathrm{He}\right)$ and ${ }^{238} \mathrm{U}(\mathrm{d}, \mathrm{p})$ surrogate reactions. The first reaction involves the fissile nucleus ${ }^{239} \mathrm{~Np}$, for which the fission and gamma-emission probabilities should sum-up to 1 below the neutron separation energy. Our data fulfill this requirement, thus validating the analysis procedure. The last three reactions involve decaying nuclei for which there is good-quality neutron-induced data to which we can compare our results. In the region where fission, neutron and gamma emission compete, we observe a good agreement between the transfer-induced and neutron-induced fission probabilities, whereas we find a very strong disagreement between the gamma-emission probabilities.

The $J^{\pi}$ distributions populated in the ${ }^{238} \mathrm{U}(\mathrm{d}, \mathrm{p})$ [5] and ${ }^{238} \mathrm{U}\left({ }^{3} \mathrm{He},{ }^{4} \mathrm{He}\right)[11]$ reactions calculated with the DWBA differ significantly from the $J^{\pi}$ distributions associated to the corresponding neutron-induced reactions. These calculations combined with our results demonstrate that fission is much less sensitive to $J^{\pi}$ differences than gamma emission. Fission and gamma-emission probabilities of ${ }^{239} \mathrm{U}$ obtained with Hauser-Feshbach calculations depend strongly on the initial spin and parity of the decaying nucleus [5]. This suggests that none of the limiting situations described in the introduction can be invoked to explain the good agreement found for the fission probabilities of ${ }^{239} \mathrm{U}$ at low excitation energy. Contrary to the calculations used for the ${ }^{239} \mathrm{U}$ case, the preliminary fission probabilities of ${ }^{237} \mathrm{U}$ obtained in [10] show a rather weak dependence on $J^{\pi}$, which explains the good agreement observed between the transfer- and neutroninduced fission probabilities of ${ }^{237} \mathrm{U}$. However, similarly to the ${ }^{239} \mathrm{U}$ case, the gamma-emission probabilities of ${ }^{237} \mathrm{U}$ are very sensitive to $J^{\pi}$. As shown in [10], it is possible to reproduce our data fairly well with the calculated $J^{\pi}$ distribution of the ${ }^{238} \mathrm{U}\left({ }^{3} \mathrm{He},{ }^{4} \mathrm{He}\right)$ reaction. This is particularly interesting, given the strong sensitivity of the gamma-emission probability to the initial $J^{\pi}$ distribution. The previous discussion shows the pertinence of our data not only for investigating the surrogate reaction-method but also for the evaluation of nuclear data [10].

We have also presented the perspectives for future measurements. In the short term we will measure the fission and gamma-emission probabilities of the even-even ${ }^{240} \mathrm{Pu}$ nucleus in direct kinematics. In the longer term we foresee measurements in inverse kinematics with radioactive beams at storage rings. These new-generation surrogate experiments should provide a wealth of high-precision fission and gamma-emission probabilities covering a broad range of nuclei. This will allow us to perform the necessary systematic studies for understanding the surrogate-reaction method and its application to regions where no neutron-induced data exists.

We would like to thank the staff of the Tandem accelerator of the IPN of Orsay for the support during the experiment and the GSI Target Laboratory for the production of the ${ }^{238} \mathrm{U}$ target. This work was supported by the French "Defi Interdisciplinaire" NEEDS of the CNRS, by the University of Bordeaux and by the European Commission within the $7^{\text {th }}$ Framework Program through CHANDA (Project No. 605203).

\section{References}

[1] J.E. Escher et al., Rev, Mod. Phys. 84, 353 (2012)

[2] G. Kessedjian et al., Phys Lett. B 692, 297 (2010)

[3] N.D. Scielzo et al., Phys. Rev. C 81, 034608 (2010)

[4] G. Boutoux et al., Phys. Lett. B 712, 319 (2012)

[5] Q. Ducasse et al., Phys. Rev. C 94, 024614 (2016)

[6] P. Marini et al., to be published

[7] G. Boutoux et al., Nucl. Instrum. Meth. A 700, 59 (2013)

[8] Q. Ducasse et al., Nucl. Instrum. Meth. A 826, 60 (2016)

[9] M.J. Lopez et al., Ann. Nucl. Energy 32, 195 (2005)

[10] O. Bouland et al., "Monte-Carlo simulation of gamma and fission transfer-induced probabilities using extended R-matrix theory: Application to the ${ }^{237} \mathrm{U}^{*}$ system", these proceedings

[11] I. Thompson, J.E. Escher, UCRL-TR-225984 (2006)

[12] B. Mei et al., Phys. Rev. C 92, 035803 (2015)

[13] M. Grieser et al., Eur. Phys. J. Special Topics 207, 1 (2012) 